\title{
The Postpartum Specific Anxiety Scale: development and preliminary validation
}

\author{
Victoria Fallon ${ }^{1}$ (D) - Jason Christian Grovenor Halford ${ }^{1} \cdot$ Kate Mary Bennett $^{1}$ • \\ Joanne Allison Harrold ${ }^{1}$
}

Received: 27 April 2016/Accepted: 12 August 2016 / Published online: 29 August 2016

(C) The Author(s) 2016. This article is published with open access at Springerlink.com

\begin{abstract}
Perinatal symptoms of anxiety are increasingly recognised due to their high prevalence and impact. Studies using pregnancy-specific anxiety measures have found that they may predict perinatal outcomes more effectively than general measures. However, no such measure exists to assess anxieties specific to the postpartum. This study aimed to develop and validate a measure (Postpartum Specific Anxiety Scale; PSAS) that accurately represents the specific anxieties faced by postpartum women, using a four-stage methodology: (1) 51 items were generated from interviews conducted with a group of 19 postpartum women at two time points, (2) the scale was reviewed and refined by a diverse expert panel, (3) an online pilot study $(n=146)$ was conducted to assess comprehensibility and acceptability and (4) an online sample of 1282 mothers of infants up to 6 months old completed the PSAS against a battery of convergent measures. A subsample $(n=262)$ repeated the PSAS 2 weeks later. The PSAS possessed good face and content validity and was comprehensible and acceptable to postpartum women. PSAS scores were significantly correlated with other measures indicating good convergent validity. Principal component analyses (PCA) revealed a simple four-factor structure. Reliability of the overall scale and individual PSAS factors proved to be good to excellent. A preliminary receiver operating characteristic (ROC) analysis also suggested that the PSAS may be a useful screening tool. The psychometric evidence suggests that the PSAS is an acceptable, valid, and reliable research tool to assess
\end{abstract}

Victoria Fallon

vfallon@liverpool.ac.uk

1 Institute of Psychology, Health and Society, University of Liverpool, Eleanor Rathbone Building, Bedford Street South, Liverpool L69 7ZA, UK anxieties, which are specific to the postpartum period. Next steps in the iterative validation process are considered for both research and screening purposes.

Keywords Postpartum $\cdot$ Anxiety $\cdot$ Maternal mental health . Scale development $\cdot$ Psychometrics

\section{Introduction}

Up to $20 \%$ of women in developed countries experience mental health problems postnatally (World Health Organisation [WHO] 2016). Several decades of research have focused on postpartum depression, while symptoms of anxiety have been largely overlooked. However, postpartum anxiety has become a condition of interest to perinatal researchers and practitioners in recognition of high prevalence rates and impact on maternal and infant outcomes (Lonstein 2007; Glasheen et al. 2010; Paul et al. 2013). Studies of postpartum anxiety reveal incidence estimates ranging from 3 to $43 \%$, with evidence suggesting that it may occur independently and at a higher rate than postpartum depression (PPD) does (Wenzel et al. 2005; Britton 2008; Glasheen et al. 2010; Paul et al. 2013).

The postpartum period involves a series of temporally unique transitions which are often experienced as stressful and overwhelming. This can lead to specific postpartum concerns such as personal appearance and postpartum weight gain (Walker and Freeland-Graves 1998), health and wellbeing of the infant (Lugina et al. 2004), interpersonal relationships (Hiser 1991) and general infant care (Warren 2005). Comprehensive reviews by Lonstein (2007) and Glasheen et al. (2010) also link postpartum anxiety to a range of adverse developmental, somatic and psychological outcomes in the infant. The interpretation of these results, however, is limited by the use of general scales of anxiety such as the State Trait Anxiety Inventory (STAI) (Spielberger 
et al. 1970) and/or scales that focus predominantly on postpartum depression (i.e. the Edinburgh Postnatal Depression Scale; EPDS; Cox et al. 1987).

General measures of anxiety are relied upon in a large majority of studies examining postpartum anxiety ${ }^{1}$ (Glasheen et al. 2010; Lonstein 2007; Meades and Ayers 2011) and may be psychometrically problematic. Many commonly used general measures include somatic items which may occur naturally in the postpartum (e.g. STAI: 'I feel rested' or 'I feel comfortable'). These may inflate anxiety scores in postpartum populations (Meades and Ayers 2011) and increase the likelihood of false positives (Swallow et al. 2003). Furthermore, symptoms of anxiety occurring in the postpartum may have distinct presentations which are not encompassed by items in general scales (Meades and Ayers 2011; Phillips et al. 2009); this limitation has been addressed when examining anxieties occurring in pregnancy (Van den Bergh 1990; Levin 1991; Wadwha et al. 1993; Huizink et al. 2002).

A variety of self-report questionnaires has been developed to assess specific anxieties relating to the gestational period that would not bear relevance in general scales. These include the Pregnancy Anxiety Scale (PAS; Levin 1991), the Pregnancy-Related Anxiety Questionnaire (PRAQ; Van Den Bergh 1990), the PRAQ-R (Huizink et al. 2004) and the Pregnancy-Related Anxiety Scale (PRAS; Wadwha et al. 1993). These measures include constructs such as fear of childbirth, foetal health and wellbeing, bearing a physically or mentally handicapped child, the mother-infant relationship, relationship changes and changes in appearance. Two key findings have been observed by studies using these measures: (a) that they predict perinatal outcomes more effectively than general measures of anxiety do (Wadwha et al. 1993; Sandman 1997) and (b) that they are qualitatively and quantitatively distinct from general indices of anxiety and depression (Huizink et al. 2002). This has led researchers to regard pregnancy-specific anxiety as a distinct entity to anxiety experienced at other times of life (Huizink et al. 2004).

In a similar manner, postpartum-specific scales have been designed to measure depression. These include the EPDS (Cox et al. 1987) and the Postpartum Depression Screening Scale (Beck and Gable 2000). Given high comorbidity with anxiety in some postpartum samples, some researchers have argued that they may be utilised to screen for both anxiety and depression concurrently (Stuart et al. 1998; Ross et al. 2003; Reck et al. 2008). While three items of the EPDS have been found to cluster together on an anxiety factor in postpartum women in several studies (Ross et al. 2003; Matthey 2008; Phillips et al. 2009; Matthey et al. 2013), the authors maintain that the scale does not measure anxiety (Cox et al. 1987). Furthermore, the EPDS does not distinguish whether anxiety

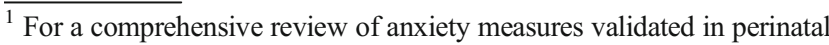
populations, refer to Meades and Ayers (2011)
}

scores on these three items are a feature of depression or a distinct entity (Matthey et al. 2003; Ross et al. 2003). This limits the utility of such tools considering work which finds that anxiety occurs more frequently (Muzik et al. 2000; Wenzel et al. 2005; Paul et al. 2013) and independently (Muzik et al. 2000; Matthey et al. 2003; Wenzel et al. 2005; Miller et al. 2006) of depression in the postpartum.

Two recent endeavours have been made to create an anxiety scale relevant to postpartum women: the Perinatal Anxiety Screening Scale (PASS; Somerville et al. 2014) and the Postpartum Worry Scale-Revised (PWS-R; Moran et al. 2014). Both measures aim to detect clinically significant levels of anxiety which map onto existing diagnostic criteria for anxiety disorders, although the PWS-R focuses only on generalised anxiety disorder (Moran et al. 2014). Emerging evidence highlights a large number of postpartum women who do not meet diagnostic criteria for an existing anxiety disorder yet experience a clinically significant degree of 'maternally focused worry' (Wenzel et al. 2005; Phillips et al. 2007; Phillips et al. 2009). As such, items within these scales may not encompass the full range of symptoms of anxiety experienced postnatally and a scale with a more focused domain is necessary. Furthermore, the PASS was designed for use with both antenatal and postnatal women (Somerville et al. 2014) suggesting that symptoms are comparable across childbirth. Although an overlap between pregnancy and postpartum anxiety has been identified (Heron et al. 2004), a body of literature provides evidence for a temporally specific pregnancy anxiety (Van den Bergh 1990; Levin 1991; Wadwha et al. 1993; Huizink et al. 2004), which includes constructs such as 'fear of childbirth' (Huizink et al. 2004) that would not be applicable postpartum. Furthermore, some women may be more prone to developing postpartum anxiety as consequence of specific physiological and psychological processes associated with birth (Heron et al. 2004), which raises additional considerations for measurement. Finally, items from both the PASS and the PWS-R were generated from researcher/ clinician experience (Moran et al. 2014; Somerville et al. 2014). Although clinicians may be the best observers of the outward manifestations of symptoms or disorder, only those who experience it can effectively capture the subjective elements (Streiner et al. 2015). This multifactorial rationale supports the development of an anxiety scale specific to the postpartum period which takes into account the limitations of the existing evidence base.

\section{Research aims}

1. To develop and validate a postpartum-specific anxiety scale 2. To investigate the structure of specific fears and worries related to the postpartum period ('postpartum anxieties') using this new scale 


\section{PSAS development}

The PSAS was developed by a doctoral student under the supervision of two experienced perinatal psychologists in the Department of Psychological Sciences at The University of Liverpool. All stages of the scale development and validation gained ethical approval from the University of Liverpool Institute of Psychology, Health and Society Ethics Committee in August 2015. All aspects of the study were performed in accordance with the 1964 Declaration of Helsinki.

\section{Stage 1: item generation}

Items were predominately informed from interviews conducted with a group of 19 postpartum women at two time points (time one 4-8 weeks; time two 12-16 weeks) to ensure that an accurate, experiential representation of postpartum-specific anxieties was achieved. Responses to the open question 'What are the main anxieties that women have at this stage of motherhood' were digitally recorded, and a basic content analysis was performed to identify themes and develop items. The scale items were further developed by reviewing validated pregnancy and postpartum anxiety questionnaires (PASS: Somerville et al. 2014; PWS-R: Moran et al. 2014; PAS: Levin 1991; PRAQ: Van Den Bergh 1990; PRAQ-R: Huizink et al. 2004; PRAS: Wadwha et al. 1993) and the postpartum anxiety research literature. The item pool was developed to systematically encompass a broad range of anxieties that were temporally specific to the postpartum period.

Consistent with other validated scales in the field, the 51item PSAS was formatted as a self-report questionnaire with a four-point Likert scale assessing the frequency of specific anxieties with consistent response options (from zero = "not at all' to three $=$ 'almost always'). The order of 27 responses was randomly reversed in order to avoid 'yea-saying' bias and aid participant concentration (Streiner et al. 2015). The wording and amount of Likert-scale divisions were chosen based on best current practice in the psychometrics literature (Streiner et al. 2015) and careful review of the self-admitted limitations of already-validated anxiety scales (Somerville et al. 2014). The timeframe for rating frequency of anxieties was limited to over the past 7 days. This is congruent with pregnancy-specific anxiety scales and deemed necessary given the transient nature of anxieties occurring in the postpartum.

\section{Stage 2: expert panel and face and content validity}

The preliminary 51 -item scale was reviewed and refined by a panel of 12 individuals, each reflecting distinct insights of scale development and/or postpartum anxiety. The panel included three experienced perinatal researchers, three senior community midwives, three research midwives (one senior), one statistician and two psychometricians. Each panel member (blind to the other members' feedback) provided detailed comments on individual items and the overall suitability of the scale. Qualitative responses from the panel indicated that the preliminary scale appeared to be measuring postpartumspecific anxieties and was both clinically acceptable for perinatal women and psychometrically feasible, indicating adequate face validity. The panel members also evaluated each item on a four-point Likert scale (four = highly relevant; three = quite relevant or highly relevant but needs rewording; two $=$ somewhat relevant; and one $=$ not relevant $).$ A content validation ratio (CVR; Streiner et al. 2015) was calculated to provide a quantitative expression of content validity. The mean CVR across all items was 0.76 which is indicative of good content validity. The panel was also asked to comment on whether any items had been omitted to further establish content coverage.

Specific qualitative feedback was collated and analysis of this phase indicated a need to revise certain aspects of the scale. Thirty-two items were reworded based on the general consensus of the panel. Of particular importance was the rewording of 11 items to reflect the specificity of postpartum anxiety. For example, the item 'I have worried about my relationship with my partner' was reworded to 'I have worried more about my relationship with my partner than before my baby was born'. Five items were deleted either due to repetition or because there was general agreement that they did not specifically relate to postpartum anxiety (e.g. low CVR). In addition, seven new items were included based on content coverage ideas provided by the panel.

The design and presentation of the final 53-item scale was then extensively reviewed to ensure it was streamlined and easy to respond to. The wording of the final items was subjected to a computer literacy check (Flesch-Kincaid Grade Level test) as being understandable for someone with 5 years of education or a 10-year-old child. A question understanding aid (QUAID) (Graesser et al. 2006) was also used, and no issues were found with the wording, syntax or semantics of the questions.

\section{Stage 3: pilot study}

An online pilot study was conducted via the Qualtrics survey software platform to assess comprehensibility of item wording, ease of responding, time taken to complete and any other implementation issues. Mothers of infants aged between 0 and 6 months $(n=146)$ were recruited via online forums (Mumsnet, Netmums) and social media platforms (Facebook groups and Twitter). Participants completed the 53-item scale and rated comprehensibility and ease of responding on two 
10-point Likert scales (i.e. 'not at all easy to understand' [0] to 'extremely easy to understand' [10] and 'not at all easy to complete' [0] to 'extremely easy to complete' [10]). An optional free text box was provided at the end of the survey to allow qualitative comments on the questionnaire content and experience of completion to be made.

The acceptability of the PSAS was excellent. The mean scores for the comprehensibility item and the ease of completion item were $9.29( \pm 1.24)$ and $9.18( \pm 1.26)$, respectively. The mean time taken to complete the 53-item scale was $9 \mathrm{~min}$ (range 3 to $15 \mathrm{~min}$ ). Based on the qualitative responses from 18 women, a 'not applicable' option was created for 7 items relating to partner, families and work to avoid response ambiguity for women who may not have these life domains. Positive comments about the scale design and items were also recorded by 36 women, which provided further evidence of its acceptability in this population.

A preliminary item analysis (endorsement frequency and item homogeneity) was also conducted on the pilot study data. The overall scale had excellent reliability (Cronbach's $\alpha=$ 0.96). Inter-item correlations were between 0.15 and 0.50 . Item-total correlations were between 0.30 and 0.70 . No problematic items were identified at this stage.

\section{Stage 4: scale reliability and validation study}

\section{Method}

\section{Measures}

Demographic information Maternal demographic questions were asked at the beginning of the online survey, including maternal age, country of residence, marital status, skill level of occupation, educational attainment, current diagnosis of anxiety and depression, timing of diagnosis and any current antidepressant/anxiety medications. Infant demographic data was also asked, including infant age, birth order, multiple birth status (twins/triplets), timing of birth and mode of feeding.

\section{The Edinburgh Postnatal Depression Scale (EPDS; Cox} et al. 1987) The EPDS is a 10-item self-report questionnaire administered to screen for depressive symptoms in the postnatal period. It is the most widely used and recommended screening scale for postnatal depression. Three items (items three, four and five) have been found to cluster together on an anxiety factor (EPDS-3A) to indicate postpartum anxiety (Matthey 2008; Matthey et al. 2013). Higher scores indicate higher levels of postpartum depressive symptoms with a score of over 10 (maximum score 30 ) indicating probable postpartum depression.
The Beck Depression Inventory-II (BDI-II; Beck et al. 1988) The BDI is a widely used self-report instrument for detecting and measuring depression. It measures the severity of 21 symptoms of general depression experienced during the past 2 weeks with higher scores indicating more severe depressive symptoms. Twenty-five years of research literature provide evidence of its reliability and validity in clinical and non-clinical samples (Beck et al. 1988).

The Spielberger State-Trait Anxiety Inventory (STAI; Spielberger et al. 1970) The STAI is a 40 -item self-report questionnaire designed to measure general anxiety. It has two separate subscales to measure situational (state) and stable (trait) anxiety. The STAI is a reliable and valid measure used with clinical and non-clinical populations and more recently in perinatal samples (Meades and Ayers 2011; Spielberger et al. 1970). Higher scores on each four-point Likert scale item indicate higher levels of anxiety.

\section{Participants}

Participants were self-identified mothers $(n=1282)$ of infants aged between birth and 6 months postpartum. The 6-month cut-off point applied reflects the complete range of theorised postpartum phases (Romano et al. 2010). Of the 1282 participants, $482(38 \%)$ were excluded from the final analyses as they had missing data on the PSAS. For full details of participation rates at each stage of the study, see Fig. 1. The age of the final sample of 800 mothers ranged from 16 to 45 years $(M=30.78 ; S D=4.96)$. The samples were predominately married (70 \%), primiparous (50\%) and professional (40\%) women from the UK $(84 \%)$. One hundred fourteen (14\%) women had a current, clinical diagnosis of anxiety/depression at the time of participation, which is comparable with UK prevalence estimates. The babies' ages ranged from 0 to 26 weeks $(M=16.20 ; S D=7.08)$ (see Table 1 for full demographic details).

\section{Procedure}

The participants were recruited through parenting forums (Mumsnet, Netmums), social media platforms (Facebook, Twitter) and other relevant websites via advertisements providing a link to the Qualtrics survey software. The advertisements stated that participants were invited to take part in a study to validate a new measure of postpartum anxiety. Prior to the main survey, an electronic consent form and information sheet were provided with a tick box to confirm that the main points had been read and understood. A single question enquired whether the participant was a mother to an infant aged between 0 and 6 months; only a positive response allowed entry to the main survey. Participants completed demographic questions followed by online versions of the 
Fig. 1 Participant flowchart

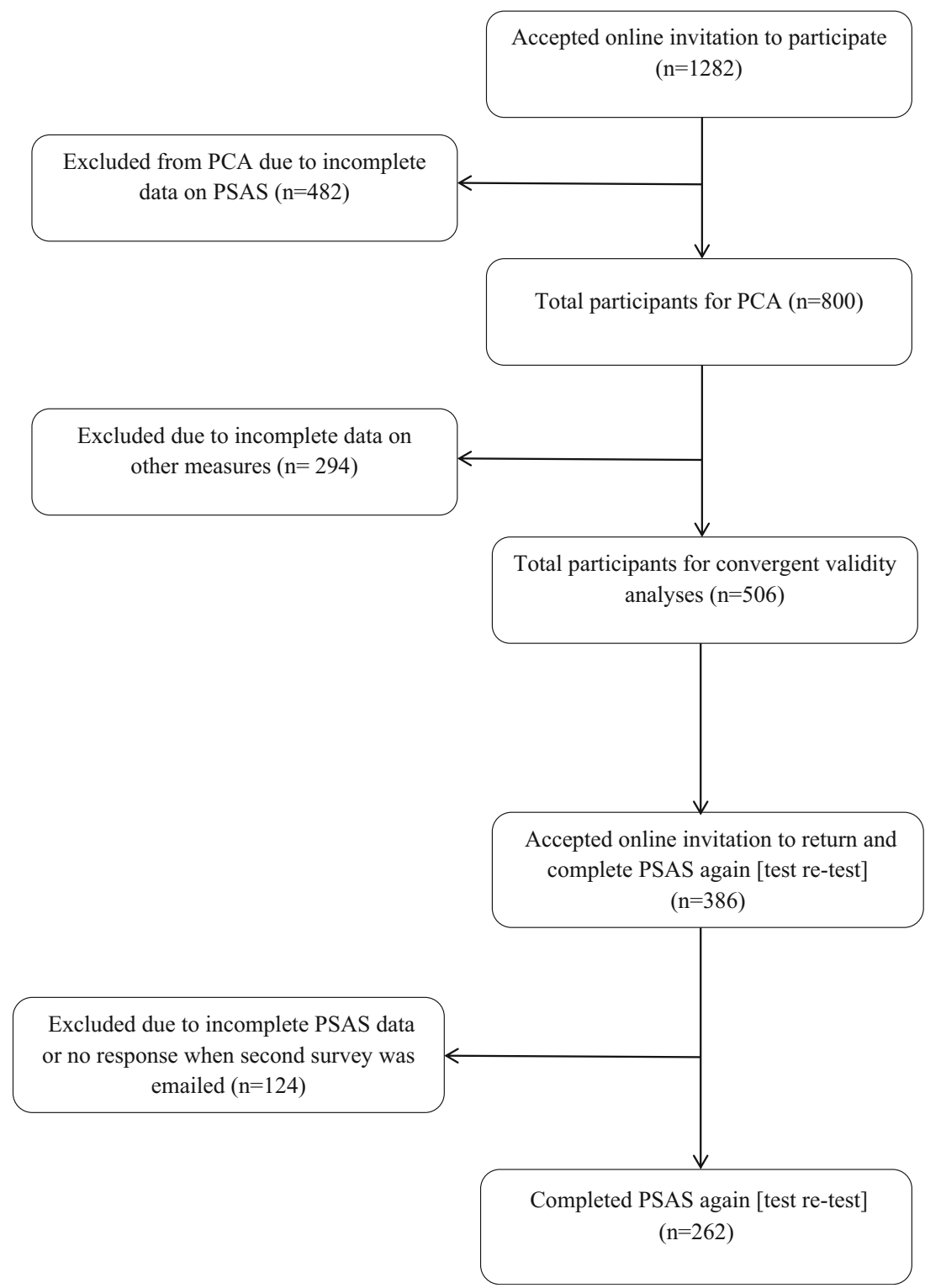

PSAS, EPDS (including EPDS-3A), BDI and STAI (state and trait). On completion of all measures, the participants were invited to return 2 weeks later to complete the PSAS again as a measure of test-retest reliability for a reimbursement of $£ 10$. Those who were willing to return received an email with the second survey containing the PSAS 2 weeks later. Responses were linked via a unique ID embedded in the survey software to preserve anonymity. Online measurement provides greater convenience and anonymity than traditional paper-based methods do (Evans and Mathur 2005) ${ }^{2}$. The potential for repetitive responding was restricted via a 'prevent ballot box stuffing' option embedded in the survey software.

\footnotetext{
${ }^{2}$ For a comprehensive review of the value of online survey methods, see Evans and Mathur (2005)
}

The online survey was accessible from April 9, 2015 to May 11, 2015.

\section{Results}

Factor structure of the PSAS

The factor structure of the PSAS was examined using data from all the participants who completed the scale $(n=800)$. A series of PCAs was conducted to determine the most appropriate number of factors to retain for rotation. Four factors were retained based on a combination of statistical tests: the results of the scree-test (Eigenvalues $>1$ and the scree plot elbow point; Cattell 1966), cumulative variance explained (highest proportion of variance while retaining the simplest, most theoretical meaningful structure; Field 2009), parallel 
Table 1 Maternal and infant demographic characteristics $(n=800)$

\begin{tabular}{|c|c|c|c|}
\hline Maternal characteristic & Value & Infant characteristic & Value \\
\hline Maternal age (mean years $\pm \mathrm{SD}$ ) & $\begin{array}{l}30.78 \\
\quad( \pm 4.96)\end{array}$ & $\begin{array}{l}\text { Infant age (mean } \\
\quad \text { weeks } \pm \mathrm{SD})\end{array}$ & $\begin{array}{l}16.20 \\
( \pm 7.08)\end{array}$ \\
\hline Country of residence $(\mathrm{n} / \%)$ & & Birth order $(n / \%)$ & \\
\hline UK and Ireland & $682(85.2)$ & First & $399(49.9)$ \\
\hline USA & $63(7.9)$ & Second & $285(35.6)$ \\
\hline Australia and NZ & $21(2.7)$ & Third & $85(10.6)$ \\
\hline Canada & $10(1.3)$ & Fourth & $19(2.4)$ \\
\hline Other European & $19(2.3)$ & Fifth and after & $12(1.5)$ \\
\hline Other non-European & $5(0.6)$ & Timing of birth $(n / \%)$ & \\
\hline Marital status $(n / \%)$ & & Premature (<37 weeks) & $38(4.7)$ \\
\hline Married & $563(70.4)$ & Early term $(>37<39)$ & $156(19.5)$ \\
\hline Cohabiting & 199 (24.9) & Full term $(>39<41)$ & $356(44.5)$ \\
\hline Single & $32(4)$ & Late term $(>41<42)$ & $141(17.6)$ \\
\hline Separated/divorced/widowed & $6(0.8)$ & Post term (>42 weeks) & $109(13.7)$ \\
\hline Occupation $(n / \%)$ & & Multiple birth $(n / \%)$ & \\
\hline Managers, directors and senior officials & $65(8.1)$ & Yes & $13(1.6)$ \\
\hline Professionals & 319 (39.9) & No & $787(98.4)$ \\
\hline Associate professional/technical & $23(2.9)$ & Mode of feeding $(n / \%)$ & \\
\hline Administrative and secretarial & $76(9.5)$ & $\begin{array}{l}\text { Exclusively } \\
\text { breastfeeding }\end{array}$ & $528(66.0)$ \\
\hline Skilled trades & $14(1.8)$ & Combination feeding & $125(15.7)$ \\
\hline Caring, leisure and other service & $91(11.4)$ & $\begin{array}{l}\text { Exclusively formula } \\
\text { feeding }\end{array}$ & $147(18.4)$ \\
\hline Sales and customer service & $70(8.8)$ & & \\
\hline Elementary occupations & $4(0.5)$ & & \\
\hline Housewife & $114(14.2)$ & & \\
\hline Not in paid occupation & $24(3.0)$ & & \\
\hline \multicolumn{4}{|l|}{ Educational attainment $\left(n / \%^{\mathrm{a}}\right)$} \\
\hline Postgraduate education & $194(24.3)$ & & \\
\hline Undergraduate education & $313(39.1)$ & & \\
\hline A-levels or equivalent college education & $169(21.1)$ & & \\
\hline GCSEs or equivalent secondary school education & $83(10.4)$ & & \\
\hline Other qualification & $27(3.4)$ & & \\
\hline No qualifications & $14(1.8)$ & & \\
\hline \multicolumn{4}{|l|}{ Current diagnosis of anxiety/depression $(n / \%)$} \\
\hline Yes & $114(14.2)$ & & \\
\hline No & $680(85.0)$ & & \\
\hline Prefer not to say & $6(0.8)$ & & \\
\hline \multicolumn{4}{|l|}{ Timing of diagnosis $\left(n / \%^{\mathrm{a}}\right)$} \\
\hline Before pregnancy & $67(58.8)$ & & \\
\hline During pregnancy & $9(1.1)$ & & \\
\hline Postpartum & $38(33.3)$ & & \\
\hline \multicolumn{4}{|l|}{$\begin{array}{l}\text { Currently prescribed medication for anxiety/depression } \\
\text { diagnosis }\left(\mathrm{N} / \%^{\mathrm{a}}\right)\end{array}$} \\
\hline Yes & $57(50)$ & & \\
\hline No & $57(50)$ & & \\
\hline
\end{tabular}

a Only participants who gave a 'yes' response to current diagnosis were included analysis (Eigenvalue that corresponds to the 95th percentile of the distribution of Eigenvalues derived from the random data;
Glorfeld 1995) and MAP test (average partial correlations between the variables after successively removing the effect 
of the factors; O'Connor 2000). This model achieved the optimal structure but revealed that seven items had factor loadings below the 0.4 threshold. Five of these items were retained ('I have felt that I should not need help to look after my baby', 'I have felt a greater need to do things in a certain way or order than before my baby was born', 'I have worried more about my finances than before my baby was born', 'I have felt that when I do get help, it is not beneficial' and 'I have worried that my baby is not developing as quickly as other babies') based on sample size requirements for practical significance (Hair et al. 1979), adequate item-total correlations ( $>0.40)$, alpha if item deleted statistics $(>0.95)$ and their theoretical relevance to postpartum anxiety, producing a 51-item scale. The PCA was conducted again, excluding the redundant items 'I have felt under pressure from health professionals to care for my baby in a certain way' and 'I have had negative thoughts about my birth experience'. Sampling adequacy for the 51-item scale was excellent $(\mathrm{KMO}=0.95)$ and Bartlett's test of sphericity demonstrated that correlations between items were large enough for PCA $\left(\chi^{2}(1275)=14,117.3, p<.001\right)$. The PCA revealed four factors, which in combination explained $44.72 \%$ of the variance.

Theoretical review of the factor loadings was conducted by two authors (VF and $\mathrm{JH}$ ) after oblique (direct oblimin) rotation (see Table 2). This revealed that factor 1 (competence and attachment anxieties) contained 15 items that addressed anxieties relating to maternal self-efficacy, parenting competence and the mother-infant relationship. Factor two (safety and welfare anxieties) had 11 items which were related to fears about infant illnesses, accidents and cot death. Factor three (practical baby care anxieties) included seven items covering anxieties that are specific to infant care such as feeding, sleeping and general routine. Finally, factor 4 (psychosocial adjustment to motherhood) contained 18 items which addressed adjustment concerns since the birth of the baby about management of personal appearance, relationships and support, work and finances and sleep.

Cross-loading items (i.e. items 14, 24, 26, 47, 49 and 51) were retained in the component with the highest loading and theoretical congruence to the other items in the factor. Item 14 ('I have felt that motherhood is much harder than expected') had similar loadings on factor 1 (competence and attachment anxieties) and factor 4 (psychosocial adjustment to motherhood). Though this item may represent difficulty adjusting, it is a competency-based question and was therefore retained in factor 1. Similarly, item 47 ('I have felt unable to juggle motherhood with other responsibilities') loaded onto factors 1 and 4. This item represented management of responsibilities and was better suited to factor 4. Items 24 ('I have worried about my baby's health even after reassurance from others') and 26 ('I have felt a greater need to do things in a certain way or order than before my baby was born') reflect the obsessivecompulsive symptoms of anxiety that are often grounded in infant safety and welfare and were retained in factor 2. Items 49 ('I have felt isolated from family and friends' and 51 ('I have felt that when I do get help it is not beneficial') both represent management of support networks and were retained in factor 4.

The four factors had excellent reliability (Cronbach's alpha ranged from 0.80 to 0.91 ; see Table 2) and had low to moderate correlations ( $r$ values ranged .26 to .39 ) indicating that they are not derived from a single underlying latent variable. The overall scale had excellent reliability (Cronbach's $\alpha=0.95$ ).

\section{Convergent validity of the PSAS}

The participants who completed all convergent and divergent measures were included in this analysis $(n=506)$. The PSAS total score was significantly correlated with theoretically related measures of anxiety (i.e. EPDS-A, STAI-state and STAItrait) and depression (i.e. EPDS, BDI) indicating good convergent validity (Table 3).

\section{Preliminary screening accuracy of the PSAS}

To preliminarily evaluate the performance of the PSAS in distinguishing between those with/without a current clinical diagnosis of anxiety/depression, a receiver operating characteristic (ROC) analysis was conducted. A statistically significant ROC curve (AUC 0.77; SE 0.02; $p<.001 ; 95 \%$ CI 0.72 , 0.81 ; Fig. 2) revealed that the optimal cut-off PSAS score for detecting clinical levels of anxiety/depression was 112 with a sensitivity and specificity of 0.75 and 0.31 , respectively. When compared to the recommended cut-off scores for the other included anxiety measures (STAI-S (45); STAI-T (45); EPDS-A (6)), the PSAS performed marginally better than the EPDS-A, which identified $73 \%$ of cases, and better than the STAI-S, which detected $63 \%$ of cases. However, it did not perform as well as the STAI-T which identified $86 \%$ of cases.

\section{Test-retest reliability of the PSAS}

Pearson correlation coefficient was calculated to examine the test-retest reliability of the PSAS for a subsample of participants $(n=262)$ who repeated the PSAS 2 weeks after the initial administration. The test-retest reliability coefficient for the PSAS was $0.88(p<.001)$, indicating excellent stability over time in the first 6 months postpartum.

\section{Discussion}

This study reports the development and initial validation of the PSAS, a 51-item measure of postpartum specific anxiety, 
Table 2 Factor structure of the PSAS (significant loadings in bold)
Rotated components

Scale item

\begin{tabular}{llll}
\hline 1 & 2 & 3 & 4
\end{tabular}

Factor 1: maternal competence and attachment anxieties 1.I have had negative thoughts about my relationship with my baby 2.I have felt that my baby would be better cared for my someone else

3.I have felt unconfident or incapable of meeting my baby's basic care needs

4.I have worried about the bond I have with my baby

5.I have worried that my baby feels more content in someone else's care 6.I have felt that other mothers are coping with their babies better than me 7.I have felt that I am not the parent I want to be

8.I have worried I will not know what to do when my baby cries

9.I have worried about how I will cope with my baby when others are not around to support me

10.I have worried about being unable to settle my baby

11.I have worried that my baby is picking up on my anxieties

12.I have worried that my baby is less content than other babies

13.I have worried that other people think my parenting skills are inadequate

14.I have felt that motherhood is much harder than expected

15.I have felt that I should not need help to look after my baby

Factor 2: infant safety and welfare anxieties

16.I have worried about my baby being accidentally harmed by someone or something else

17.I have repeatedly checked on my sleeping baby

18.I have worried that my baby will stop breathing while sleeping

19.I have felt frightened when my baby is not with me

20.I have worried about leaving my baby in a childcare setting

21.I have worried about accidentally harming my baby

22.I have thought of ways to avoid exposing my baby to germs

23.I have not taken part in an everyday activity with my baby because I fear they may come to harm

24.I have worried about my baby's health even after reassurance from others

25.I have worried that I will become too ill to care for my baby

26.I have felt a greater need to do things in a certain way or order than before my baby was born

Factor 3: practical infant care anxieties

27.I have worried about my baby's milk intake

28.I have worried about my baby's weight

29.I have worried about getting my baby into a routine

30.I have worried about the way that I feed my baby

31.I have worried about the length of time that my baby sleeps

32.I have used the internet for reassurance about my baby's health

33.I have worried that my baby is not developing as quickly as other babies

Factor 4: psychosocial adjustment to motherhood

34.I have felt resentment towards my partner

35.I have felt tired even after a good amount of rest

36.I have worried more about my relationship with my partner than before my baby was born

37.I have worried that I am not going to get enough sleep

38.I have worried that my partner finds me less attractive than before my baby was born

$\begin{array}{llll}\mathbf{0 . 7 3} & -0.06 & 0.08 & 0.06 \\ \mathbf{0 . 7 2} & 0.01 & 0.03 & -0.04 \\ \mathbf{0 . 6 6} & 0.10 & 0.20 & -0.07 \\ \mathbf{0 . 6 6} & 0.050 . & 0.12 & 0.07 \\ \mathbf{0 . 6 2} & 0.21 & 0.02 & -0.05 \\ \mathbf{0 . 5 9} & -0.01 & 0.22 & 0.20 \\ \mathbf{0 . 5 7} & -0.04 & -0.03 & 0.31 \\ \mathbf{0 . 5 4} & 0.11 & 0.24 & 0.01 \\ \mathbf{0 . 5 3} & 0.09 & 0.08 & 0.08 \\ \mathbf{0 . 5 2} & -0.05 & 0.36 & 0.02 \\ \mathbf{0 . 4 9} & 0.13 & 0.02 & 0.27 \\ \mathbf{0 . 4 7} & -0.05 & 0.42 & -0.01 \\ \mathbf{0 . 4 1} & 0.18 & 0.08 & 0.31 \\ & & & \\ \mathbf{0 . 4 1} & -0.16 & 0.17 & 0.40 \\ \mathbf{0 . 3 6} & 0.09 & -0.06 & 0.26 \\ & & & \\ 0.12 & \mathbf{0 . 7 6} & -0.02 & -0.01 \\ & & & \\ -0.05 & \mathbf{0 . 7 1} & 0.05 & 0.02 \\ -0.02 & \mathbf{0 . 6 8} & 0.11 & -0.02 \\ 0.03 & \mathbf{0 . 6 7} & -0.09 & 0.19 \\ -0.12 & \mathbf{0 . 5 5} & 0.03 & 0.28 \\ 0.27 & \mathbf{0 . 5 2} & 0.00 & -0.07 \\ -0.12 & \mathbf{0 . 5 1} & 0.17 & 0.02 \\ 0.29 & \mathbf{0 . 4 8} & -0.09 & 0.10 \\ 0.16 & \mathbf{0 . 4 8} & 0.42 & -0.02 \\ 0.30 & \mathbf{0 . 4 3} & 0.08 & 0.02 \\ 0.02 & \mathbf{0 . 2 9} & 0.13 & 0.28\end{array}$

$\begin{array}{llll}-0.01 & 0.05 & \mathbf{0 . 7 4} & -0.04\end{array}$

$\begin{array}{llll}0.07 & 0.12 & \mathbf{0 . 6 8} & -0.12\end{array}$

$\begin{array}{llll}0.08 & -0.09 & \mathbf{0 . 6 7} & 0.14\end{array}$

$\begin{array}{llll}0.15 & 0.07 & \mathbf{0 . 6 2} & 0.00\end{array}$

$\begin{array}{llll}0.10 & -0.18 & \mathbf{0 . 5 4} & 0.26\end{array}$

$\begin{array}{llll}0.00 & 0.27 & \mathbf{0 . 4 4} & 0.08\end{array}$

$\begin{array}{llll}0.25 & 0.19 & \mathbf{0 . 3 2} & 0.05\end{array}$

$\begin{array}{llll}0.05 & -0.09 & 0.04 & \mathbf{0 . 5 9}\end{array}$

$\begin{array}{llll}0.07 & 0.05 & -0.03 & \mathbf{0 . 5 8}\end{array}$

$\begin{array}{llll}0.11 & 0.16 & -0.07 & \mathbf{0 . 5 7}\end{array}$

$\begin{array}{llll}0.07 & -0.23 & 0.23 & \mathbf{0 . 5 6}\end{array}$

$\begin{array}{llll}-0.13 & 0.16 & 0.11 & \mathbf{0 . 5 6}\end{array}$ 
Table 2 (continued)

\begin{tabular}{|c|c|c|c|c|}
\hline \multirow[b]{2}{*}{ Scale item } & \multicolumn{4}{|c|}{ Rotated components } \\
\hline & 1 & 2 & 3 & 4 \\
\hline $\begin{array}{l}\text { 39.I have worried more about my relationship with my family than before } \\
\text { my baby was born }\end{array}$ & 0.13 & 0.04 & -0.12 & 0.54 \\
\hline $\begin{array}{l}\text { 40.I have worried more about my appearance than before my baby was } \\
\text { born }\end{array}$ & -0.26 & 0.06 & 0.10 & $\mathbf{0 . 5 5}$ \\
\hline $\begin{array}{l}\text { 41.I have worried more about completing household chores than before } \\
\text { my baby was born }\end{array}$ & 0.03 & 0.02 & 0.23 & 0.52 \\
\hline 42.I have had difficulty sleeping even when I have had the chance to & 0.01 & 0.20 & 0.01 & 0.51 \\
\hline 43.I have felt that I do not get enough support & 0.26 & 0.01 & -0.01 & 0.49 \\
\hline $\begin{array}{l}\text { 44.I have worried more about my relationship with my friends than before } \\
\text { my baby was born }\end{array}$ & 0.16 & 0.14 & -0.05 & 0.48 \\
\hline $\begin{array}{l}\text { 45.I have been less able to concentrate on simple tasks than before my } \\
\text { baby was born }\end{array}$ & 0.25 & 0.07 & -0.01 & 0.47 \\
\hline 46.I have worried about returning to work & -0.18 & 0.27 & 0.07 & 0.46 \\
\hline 47.I have felt unable to juggle motherhood with other responsibilities & 0.38 & -0.07 & -0.13 & 0.45 \\
\hline $\begin{array}{l}\text { 48.I have felt that I have had less control over my day than before my baby } \\
\text { was born }\end{array}$ & 0.25 & -0.09 & 0.20 & 0.43 \\
\hline 49.I have felt isolated from family and friends & 0.35 & 0.18 & -0.12 & 0.40 \\
\hline 50.I have worried more about my finances than before my baby was born & -0.11 & 0.22 & 0.17 & $\mathbf{0 . 3 5}$ \\
\hline 51.I have felt that when I do get help it is not beneficial & 0.25 & 0.27 & 0.01 & 0.31 \\
\hline$\%$ of variance explained & 29.94 & 6.35 & 4.84 & 3.56 \\
\hline Cronbach's alpha & 0.91 & 0.85 & 0.80 & 0.90 \\
\hline
\end{tabular}

using a large online sample of mothers in the first 6 months postpartum. The results suggest that the PSAS is an acceptable measure with sound psychometric properties. The low to moderate size correlations between factors indicated that they are not derived from a single underlying latent variable. It has a simple four-factor structure which showed good face and content validity and can be distinguished as (1) competence and attachment anxieties, (2) infant safety and welfare anxieties, (3) practical baby care anxieties and (4) psychosocial adjustment to motherhood.

Despite limited discussion about the qualitative nature of the symptoms of postpartum anxiety, these constructs are theoretically meaningful when examined in relation to some recent work. Brockington et al. (2006) found qualitative themes of 'fear of cot death', 'fear of the criticism of mothering skills' and 'fear of disordered maternal attachment' in a sample of 129 women referred to psychiatric services. Similar symptoms were also found in a recent interview study (Highet et al. 2014) alongside a theme of 'adjustment difficulties' which included anxieties relating to changes in appearance, daily activities and social roles. Phillips et al. (2009) investigated

Table 3 Pearson product-moment correlations between the PSAS and other validated measures of anxiety and depression $(n=506)$

\begin{tabular}{llllll}
\hline & BDI & STAI-state & STAI-trait & EPDS & EPDS-A \\
\hline PSAS & $0.76^{*}$ & $0.74 *$ & $0.77^{*}$ & $0.81^{*}$ & $0.75^{*}$ \\
\hline
\end{tabular}

$* p<.01$ (one tailed) symptom presentations of postpartum women with an anxiety disorder not otherwise specified (ADNOS). They identified $65 \%$ of women reporting anxieties in relation to infant health, safety and wellbeing; $53 \%$ with anxieties concerning performance as a mother; and $18 \%$ with anxieties relating to practical day-to-day care of the infant. This finding suggests that the PSAS, unlike existing measures, may possess constructs

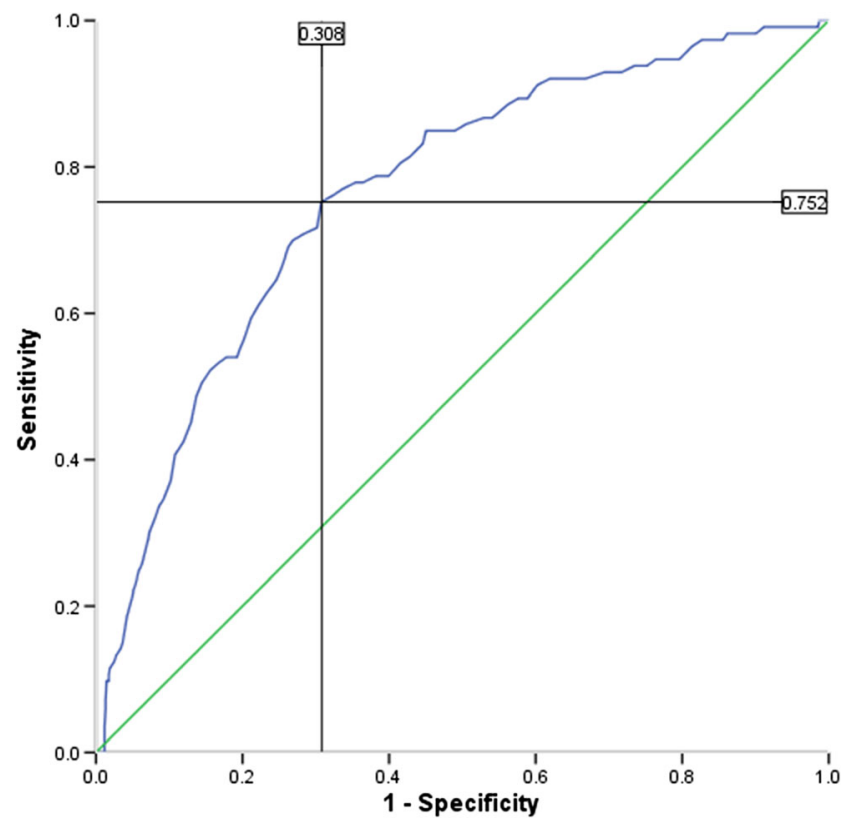

Fig. 2 Receiver operating characteristic (ROC) curve analysis. Area under the curve 0.77 
that are sensitive to postpartum women experiencing clinically significant maternally focused worry, yet failing to meet diagnostic criteria for an anxiety disorder (Phillips et al. 2009). Further examination of the construct validity of the PSAS is necessary to reexamine the proposed model and to provide further confirmation of these factors.

As hypothesised, the PSAS was significantly positively correlated with theoretically related measures of anxiety, which demonstrates initial evidence of convergent validity. The PSAS was also significantly associated with measures of depression, which was anticipated given the high comorbidity identified in previous work (Stuart et al. 1998; Ross et al. 2003; Reck et al. 2008) and provides further convergent support. It has been suggested that the overlap between depression and anxiety reflects the co-occurrence of phenomenologically distinct constructs (Beck 1976; Beck et al. 1979; Burns and Eidelson 1998). As such, Burns and Eidelson (1998) contend that any valid and reliable measure of anxiety and depression should correlate approximately at the 0.70 level; the PSAS exceeded this benchmark. In addition, the internal consistency of the overall PSAS scale and four factors was good to excellent (George and Mallery 2003; Ponterotto and Ruckdeschel 2007). Test-retest reliability also indicated better stability over time than other recent endeavours (Somerville et al. 2014).

A preliminary ROC analysis demonstrated that the PSAS performed well at identifying women with a current clinical diagnosis of anxiety and/or depression. At the optimal cut-off score of $112,75 \%$ of women with a diagnosis were detected, which surpasses other recent efforts (Somerville et al. 2014). Furthermore, the PSAS performed better than did other general (i.e. STAI-S) and postpartum-specific (i.e. EPDS-A) measures of anxiety. However, determining the case finding abilities of the PSAS was not a primary aim of the research and it is acknowledged that the self-report methods used to ascertain a current, clinical diagnosis of anxiety and/or depression in the sample are crude compared to other work (Somerville et al. 2014). Furthermore, the design precluded the differentiation of anxiety and depression within the sample. Interestingly, trait anxiety had the best case-finding abilities and previous work has suggested that the trait scale may examine depression, as well as anxiety (Bieling et al. 1998; Julian 2011), which could explain the high area under the curve (AUC) observed in this sample. Despite these limitations, the analysis suggests that the PSAS may be a useful screening tool for postpartum women and future work in clinical samples across the full spectrum of anxiety disorders is necessary to confirm this.

In the interim, the PSAS can be used to capture a range of anxieties relating to both mother and infant, which are specific to the postpartum period. Other potential avenues for research use include examining the prevalence of postpartum-specific anxiety and examining how this varies in different populations (e.g. those with high-risk pregnancies, mothers of premature infants, mothers who have experienced previous miscarriage or stillbirth). Administering the PSAS in samples of postpartum women with non-comorbid anxiety and depression will allow examination of whether the PSAS measures 'pure' anxiety and can differentiate anxiety from depression. A comparison of scores on the PSAS in women with ADNOS and other anxiety disorders (e.g. GAD, OCD) would be particularly interesting given recent findings concerning maternally focused worry in samples of postpartum women with ADNOS (Phillips et al. 2007; Phillips et al. 2009).

Validation of a measure is an iterative process and there are several areas for future work that are necessary to continue the development and evaluation of the PSAS. Firstly, the study used an online convenience sample which provided an appropriate sample size for the analyses conducted (in particular PCA) but lacked sampling control. The samples were predominately married, professional women from the UK. Thus, the psychometric properties of the PSAS may vary in other populations, and it will be important to replicate these findings in diverse samples, particularly those at risk of developing postpartum anxiety. Second, the pilot study demonstrated excellent acceptability to postpartum women in its current form, which probably reflects the qualitative inquiry used to inform its development. However, the item analyses (inter-item, item total) displayed psychometric potential for the development of a short form, which may increase its utility in both clinical and research settings.

Finally, the pregnancy anxiety literature provides findings that differentiate pregnancy-specific anxiety from general measures of anxiety and depression (Huizink et al. 2004) and highlights that temporally specific measures may be more efficacious at predicting perinatal outcomes than the more commonly used general measures (Wadwha et al. 1993; Huizink et al. 2002; Huizink et al. 2003). Further research should attempt to replicate this work with the PSAS. Isolation of child-bearing-related anxiety from symptoms of general anxiety and depression may allow clinicians and researchers to address issues of identification, prediction and prevention more precisely (Huizink et al. 2004). Associations between postpartum anxiety and maternal attachment (Mertesacker et al. 2004), infant feeding (Britton 2007; Paul et al. 2013) and infant temperament (Coplan et al. 2005) have been previously identified and warrant examination to ascertain the predictive value of the PSAS for maternal and infant outcomes and determine whether it may be a more effective predictor of perinatal outcomes than general measures of anxiety.

Compliance with ethical standards The PSAS was developed by a doctoral student under the supervision of two experienced perinatal psychologists in the Department of Psychological Sciences at The University of Liverpool. All stages of the scale development and validation gained 
ethical approval from the University of Liverpool Institute of Psychology, Health and Society Ethics Committee in August 2015. All aspects of the study were performed in accordance with the 1964 Declaration of Helsinki.

Conflict of interest The authors declare that they have no conflicts of interest.

Funding The authors have no financial relationships relevant to this article to disclose.

Open Access This article is distributed under the terms of the Creative Commons Attribution 4.0 International License (http:// creativecommons.org/licenses/by/4.0/), which permits unrestricted use, distribution, and reproduction in any medium, provided you give appropriate credit to the original author(s) and the source, provide a link to the Creative Commons license, and indicate if changes were made.

\section{References}

Beck AT (1976) Cognitive therapy and the emotional disorders. International Universities Press, New York

Beck C, Gable R (2000) Postpartum Depression Screening Scale: development and psychometric testing. Nurs Res 49:272-282

Beck AT, Rush A, Shaw B, Emery G (1979) Cognitive therapy of depression. Guildford Press, New York

Beck AT, Steer RA, Carbin MG (1988) Psychometric properties of the Beck Depression Inventory: twenty-five years of evaluation. Clin Psychol Rev 8:77-100. doi:10.1016/0272-7358(88)90050-5

Bieling PJ, Antony MM, Swinson RP (1998) The state-trait anxiety inventory, trait version: structure and content re-examined. Behav Res Ther 36:777-788. doi:10.1016/S0005-7967(98)00023-0

Britton JR (2007) Postpartum anxiety and breastfeeding. J Reprod Med 52:689-695

Britton JR (2008) Maternal anxiety: course and antecedents during the early postpartum period. Depress Anxiety 25:793-800. doi:10.1002 /da.20325

Brockington IF, Macdonald E, Wainscott G (2006) Anxiety, obsessions and morbid preoccupations in pregnancy and the puerperium. Arch Womens Ment Health 9:253-263. doi:10.1007/s00737-006-0134-Z

Burns D, Eidelson R (1998) Why are depression and anxiety correlated? a test of the tripartite model. J Consult Clin Psychol 66:461-473

Cattell R (1966) The scree test for the number of factors. Multivar Behav Res 1:245-276

Coplan RJ, O’Neil K, Arbeau K (2005) Maternal anxiety during and after pregnancy and infant temperament at three months of age. J Prenat Perinat Psychol Heal 19:199-215, doi:10.1108/17506200710779521

Cox JL, Holden JM, Sagovsky R (1987) Detection of postnatal depression: development of the 10-item Edinburgh Postnatal Depression scale. Br J Psychiatry 150:782-786. doi:10.1192/bjp.150.6.782

Evans JR, Mathur A (2005) The value of online surveys. Internet Res 15: 195-219. doi:10.1108/10662240510590360

Field A (2009) Discovering statistics using SPSS. Sage Publications, London

George D, Mallery M (2003) Using SPSS for Windows step by step: a simple guide and reference. Allyn and Bacon, Boston, MA

Glasheen C, Richardson G, Fabio A (2010) A systematic review of the effects of postnatal maternal anxiety on children. Arch Womens Ment Health 13:61-74. doi:10.1007/s00737-009-0109-y

Glorfeld L (1995) An improvement on Horn's parallel analysis methodology for selecting the correct number of factors to retain. Educ Psychol Meas 55:377-393
Graesser AC, Cai Z, Louwerse MM, Daniel F (2006) Question Understanding Aid (QUAID) - a Web facility that tests question comprehensibility. Public Opin Q 70:3-22. doi:10.1093/poq/nfj012

Hair J, Anderson R, Tatham R, Grablowsky B (1979) Multivariate data analysis. Pipe Books, Tulsa, Oklahoma

Heron J, O'Connor TG, Evans J et al (2004) The course of anxiety and depression through pregnancy and the postpartum in a community sample. J Affect Disord 80:65-73. doi:10.1016/j.jad.2003.08.004

Highet N, Stevenson AL, Purtell C, Coo S (2014) Qualitative insights into women's personal experiences of perinatal depression and anxiety. Women Birth 27:179-184. doi:10.1016/j.wombi.2014.05.003

Hiser P (1991) Maternal concerns during the early postpartum. J Am Assoc Nurse Pract 3(4):166-173

Huizink AC, Robles De Medina PG, Mulder EJH et al (2002) Psychological measures of prenatal stress as predictors of infant temperament. J Am Acad Child Adolesc Psychiatry 41:10781085. doi:10.1097/00004583-200209000-00008

Huizink AC, Robles de Medina PG, Mulder EJH et al (2003) Stress during pregnancy is associated with developmental outcome in infancy. J Child Psychol Psychiatry 44:810-8

Huizink AC, Mulder EJH, Robles de Medina PG et al (2004) Is pregnancy anxiety a distinctive syndrome? Early Hum Dev 79:81-91. doi:10.1016/j.earlhumdev.2004.04.014

Julian LJ (2011) Measures of anxiety. Arthritis Care Res 63:0-11. doi:10.1002/acr.20561.Measures

Levin JS (1991) The factor structure of the Pregnancy Anxiety scale. J Heal Soc Behav 32:368-381

Lonstein JS (2007) Regulation of anxiety during the postpartum period. Front Neuroendocrinol 28:115-141. doi:10.1016/j. yfrne.2007.05.002

Lugina HI, Nystrom L, Christensson K, Lindmark G (2004) Assessing mothers' concerns in the postpartum period : methodological issues. J Adv Nurs 48(3):279-290

Matthey S (2008) Using the Edinburgh Postnatal Depression scale to screen for anxiety disorders. Depress Anxiety 25:926-931. doi: $10.1002 /$ da. 20415

Matthey S, Barnett B, Howie P, Kavanagh DJ (2003) Diagnosing postpartum depression in mothers and fathers: whatever happened to anxiety? J Affect Disord 74:139-147. doi:10.1016/S0165-0327(02 )00012-5

Matthey S, Fisher J, Rowe H (2013) Using the Edinburgh postnatal depression scale to screen for anxiety disorders: conceptual and methodological considerations. J Affect Disord 146:224-230. doi:10.1016/j.jad.2012.09.009

Meades R, Ayers S (2011) Anxiety measures validated in perinatal populations: a systematic review. J Affect Disord 133:1-15. doi:10. 1016/j.jad.2010.10.009

Mertesacker B, Bade U, Haverkock A, Pauli-Pott U (2004) Predicting maternal reactivity/sensitivity: the role of infant emotionality, maternal depressiveness/anxiety, and social support. Infant Ment Health J 25:47-61. doi:10.1002/imhj.10085

Miller RL, Pallant JF, Negri LM (2006) Anxiety and stress in the postpartum: is there more to postnatal distress than depression? BMC Psychiatry 6:12. doi:10.1186/1471-244X-6-12

Moran TE, Polanin JR, Wenzel A (2014) The postpartum worry scalerevised: an initial validation of a measure of postpartum worry. Arch Womens Ment Health 17:41-48. doi:10.1007/s00737-013-0380-9

Muzik M, Klier CM, Rosenblum KL et al (2000) Are commonly used self-report inventories suitable for screening postpartum depression and anxiety disorders? Acta Psychiatr Scand 102:71-3. doi:10. 1034/j.1600-0447.2000.102001071.x

O'Connor B (2000) SPSS and SAS programs for determining the number of components using Parallel Analysis and Velicer's MAP test. Behav Res Methods, Instruments Comput 1:396-402. doi:10.1017 /CBO9781107415324.004 
Paul IM, Downs DS, Schaefer EW et al (2013) Postpartum anxiety and maternal-infant health outcomes. Pediatrics 131:e1218-24. doi:10.1542/peds.2012-2147

Phillips J, Sharpe L, Matthey S (2007) Rates of depressive and anxiety disorders in a residential mother-infant unit for unsettled infants. Aust N Z J Psychiatry 41:836-842. doi:10.1080/00048670701579108

Phillips J, Sharpe L, Matthey S, Charles M (2009) Maternally focused worry. Arch Womens Ment Health 12:409-418. doi:10. 1007 /s00737-009-0091-4

Ponterotto J, Ruckdeschel D (2007) An overview of coefficient alpha and a reliability matrix for estimating adequacy of internal consistency coefficient with psychological research measures. Percept Mot Skills 105:997-1014

Reck C, Struben K, Backenstrass M et al (2008) Prevalence, onset and comorbidity of postpartum anxiety and depressive disorders. Acta Psychiatr Scand 118:459-468. doi:10.1111/j.1600-0447.2008.01264.x

Romano M, Cacciatore A, Giordano R, La Rosa B (2010) Postpartum period: three distinct but continuous phases. J Prenat Med 4:22-25

Ross LE, Evans SEG, Sellers EM, Romach MK (2003) Measurement issues in postpartum depression part 1: anxiety as a feature of postpartum depression. Arch Womens Ment Health 6:51-57. doi:10.1007/s00737-002-0155-1

Sandman CA, Wadwha PD, Chicz-Demet A, Dunkel-Schetter C, Porto M (1997) Maternal stress, HPA activity, and fetal/infant outcome. Ann N Y Acad Sci 814:266-275

Somerville S, Dedman K, Hagan R, et al (2014) The Perinatal Anxiety Screening Scale: development and preliminary validation. Arch Womens Ment Health 443-454. doi: 10.1007 /s00737-014-0425-8
Spielberger C, Gorsuch R, Lushene R (1970) Manual for the State-Trait Anxiety Inventory. Consultant Psychologists Press, Palo Alto (CA)

Streiner D, Norman G, Cairney J (2015) Health Measurement Scales: a practical guide to their development and use, 5th edn. Oxford University Press, London

Stuart S, Couser G, Schilder K et al (1998) Postpartum anxiety and depression: onset and comorbidity in a community sample. J Nerv Ment Dis 186:420-424

Swallow B, Lindow S, Masson E, Hay D (2003) The use of the General Health Questionnaire (GHQ-28) to estimate prevalence of psychiatric disorder in early pregnancy. Psychol Heal Med $8: 213-217$

Van den Bergh B (1990) The influence of maternal emotions during pregnancy on fetal and neonatal behavior publication date. $\mathrm{J}$ Prenat Perinat Psychol Heal 5(2):119-130

Walker L, Freeland-Graves J (1998) Lifestyle factors related to postpartum weight gain and body image in bottle- and breastfeeding women. J Obstet Gynecol Neonatal Nurs 27(2):151-160

Wadwha PD, Sandman CA, Porto M et al (1993) The association between prenatal stress and infant birth weight and gestational age at birth: a prospective investigation. Am J Obstet Gynecol 169:858-865

Warren PL (2005) First-time mothers: social support and confidence in infant care. J Adv Nurs 50:479-488. doi:10.1111/j.13652648.2005.03425.x

Wenzel A, Haugen EN, Jackson LC, Brendle JR (2005) Anxiety symptoms and disorders at eight weeks postpartum. J Anxiety Disord 19: 295-311. doi:10.1016/j.janxdis.2004.04.001

WHO (2016) Gender and women's mental health. Retrieved January 10, 2016, from http://www.who.int/mental_health/prevention/ genderwomen/en/ 